\title{
VANISHING FOR HODGE IDEALS ON TORIC VARIETIES
}

\author{
YAJNASENI DUTTA
}

\begin{abstract}
In this article we construct a Koszul-type resolution of the $p^{\text {th }}$ exterior power of the sheaf of holomorphic differential forms on smooth toric varieties and use this to prove a Nadel-type vanishing theorem for Hodge ideals associated to effective $\mathbb{Q}$-divisors on smooth projective toric varieties. This extends earlier results of Mustaţă and Popa.
\end{abstract}

\section{INTRODUCTION}

In this paper we prove a Nadel-type vanishing statement for Hodge ideals on smooth projective toric varieties. This extends similar vanishing statements in [MP16] and [MP18].

In a series of papers, Mustaţă and Popa thoroughly studied Hodge ideals with the goal of understanding singularities and Hodge theoretic properties of hypersurfaces in smooth varieties. These ideals are indexed by non-negative integers, with the $0^{\text {th }}$ being a multiplier ideal (see Remark 1.4 below). In particular, a hypersurface $D$ in a smooth variety $X$ has log canonical singularities if and only if $I_{0}(D) \simeq \mathcal{O}_{X}$. For the sake of applications it is fundamental for such gadgets to satisfy vanishing theorems, just like multiplier ideals.

To formulate our statement, let $X$ be a smooth projective toric variety of dimension $n$, with torus invariant divisors $D_{i}, i=1, \cdots, d$. Let $D$ be an effective $\mathbb{Q}$-divisor on $X$ such that there is an integer $\ell$ and a section $s$ of some line bundle $M^{\otimes \ell}$ satisfying $D=\frac{1}{\ell} H$ where $H=(s=0)$. Then we have the following

Definition 1.1 (Property $P_{k}(D)$ ). With the above assumption on $D$, we say that a line bundle $L$ on a smooth toric variety $X$, satisfies property $P_{k}(D)$ if the divisors $L+D_{\text {red }}-D$ and $L+D_{\tau_{1}}+\cdots+D_{\tau_{p}}+D_{\text {red }}-D$ for all $1 \leq p \leq k$ and for all $\tau_{i} \in\{1, \cdots, d\}$ are ample. Moreover, when $D$ and $D_{i}$ 's are ample, we also allow $L \sim_{\mathbb{Q}} D-D_{\text {red }}$.

Remark/Notation 1.2. When $D$ is reduced, $D=D_{\text {red }}$ and therefore Property $P_{k}(D)$ does not depend on $D$. In this case, we will use the notation $P_{k}$ to emphasise the lack of dependence on $D$.

Theorem A. With the notation as above, for a fixed integer $k$ and a line bundle $L$ on $X$ satisfying property $P_{k}(D)$ we have

$$
H^{i}\left(X, \omega_{X}\left((k+1) D_{r e d}\right) \otimes L \otimes I_{k}(D)\right)=0 \text { for all } i>0 .
$$

In [MP16, MP18] Mustaţă and Popa showed such vanishing statements in general, for smooth projective varieties, as well as for projective spaces and for abelian varieties with much weaker hypotheses (see [MP16, Theorem 25.3] and [MP18, Variant 12.5, Remark 12.4]). However their general statement required additional hypotheses. For instance, on smooth projective toric varieties, they require $D$ to be reduced $(k-1)$ - $\log$ canonical (i.e. $I_{p}(D) \simeq \mathcal{O}_{X}\left(D_{\text {red }}-\lceil D\rceil\right)$ for all $p \leq k-1$ ) and the ample line bundle $L$ to satisfy certain positivity properties with respect to $D$ (see [MP16, Corollary 25.1]). Here we only need to assume certain positivity properties, depending essentially only on the toric data, on the line bundle $L$. Our statement recovers the statement for projective spaces as we will see in Example 1.

2010 Mathematics Subject Classification. Primary 14F17; Secondary 14J17, 32S25.

Key words and phrases. Nadel vanishing, Hodge ideals, Toric varieties. 
Remark 1.3. The assumption that $\ell D \sim M^{\otimes \ell}$ is not necessary to define the Hodge ideal, but it is to ensure the gluing of the local $\mathcal{D}$-modules that are used to define the Hodge ideals for $\mathbb{Q}$-divisors (see Remark 2.4 for more details). Existence of such global objects is crucial for the purpose of the proof we present. Note that such assumptions can always be realised on a finite flat modification of $X$ and $D$.

Remark 1.4. We know from [MP16, Proposition 10.1] (or in the $\mathbb{Q}$-divisor setting from [MP18, Proposition 9.1]) that $I_{0}(D) \simeq \mathcal{J}((1-\epsilon) D)$, the multiplier ideal sheaf of $(1-\epsilon) D$ for $0<\epsilon \ll 1$. Note that when $k=0$, Theorem $\mathrm{A}$ is the same as Nadel vanishing for this multiplier ideal sheaf.

On a smooth toric variety $X$ of dimension $n$, we have the short exact sequence (see [CLS11, Theorem 8.1.6])

$$
0 \rightarrow \Omega_{X}^{1} \rightarrow \bigoplus_{i=1}^{d} \mathcal{O}_{X}\left(-D_{i}\right) \rightarrow \mathcal{O}_{X}^{\oplus d-n} \rightarrow 0
$$

where the $D_{i}$ 's are the torus invariant divisors of $X$. Using this Euler type short exact sequence, we construct a resolution of the $k^{\text {th }}$ exterior power of the sheaf of holomorphic differential forms $\Omega_{X}^{k}$ on $X$ (see Lemma 2.2). Then an inductive argument, similar to that in [MP16, Theorem 25.3] for projective spaces, yields the above vanishing statement. We first look at some examples:

\section{Examples.}

(1) Projective spaces: When $X=\mathbb{P}^{n}$ and $\operatorname{deg} D=d$, our statement recovers the statement in [MP16, Theorem 15.3](Or, [MP18, Variant 12.5]), namely

$$
H^{i}\left(X, \mathcal{O}_{X}(\ell) \otimes \mathcal{O}_{X}\left(k D_{\text {red }}\right) \otimes I_{k}(D)\right)=0 \text { for all } i>0
$$

and $\ell \geq d-n-1$. Indeed, torus invariant divisors on $\mathbb{P}^{n}$ satisfy $\mathcal{O}_{\mathbb{P}^{n}}\left(D_{i}\right) \simeq \mathcal{O}_{\mathbb{P}^{n}}(1)$ and therefore are ample. In the above statement taking $\mathcal{O}_{X}(\ell) \simeq \omega_{X} \otimes L$, we see that when $\ell \geq d-n-1, L-D_{\text {red }}$ satisfies property $P_{k}(D)$.

(2) Products of projective spaces: When $X=\mathbb{P}^{n_{1}} \times \mathbb{P}^{n_{2}}$, and $D$ is an effective $\mathbb{Q}$-divisor in the class $(c, d)$, we have

$$
H^{i}\left(X, \mathcal{O}_{X}(a, b) \otimes \mathcal{O}_{X}\left(k D_{\text {red }}\right) \otimes I_{k}(D)\right)=0 \text { for all } i>0
$$

whenever $a \geq c-n_{1}$ and $b \geq d-n_{2}$. Indeed, the torus invariant divisors are all of type $(1,0)$ or $(0,1)$ and therefore nef. Then a line bundle $L \simeq \mathcal{O}_{X}\left(a^{\prime}, b^{\prime}\right) L-D_{\text {red }}$ satisfies property $P_{k}(D)$ if $a^{\prime} \geq c+1$ and $b^{\prime} \geq d+1$. It is worth mentioning that when $D$ is reduced, we get

$$
H^{i}\left(X, \mathcal{O}_{X}\left((k+1) c-n_{1},(k+1) d-n_{2}\right) \otimes I_{k}(D)\right)=0 \text { for all } i>0 .
$$

(3) Hirzebruch surfaces: When $X=F_{r}=\mathbb{P}(\mathcal{O} \oplus \mathcal{O}(r))$, the Hirzebruch surface with $r \geq 0$, we have Pic $X=\mathbb{Z} F \oplus \mathbb{Z} E$, where $E \sim E^{\prime}+r F, E^{\prime}$ is the class of the section of self-intersection $-r$ and $F$ is the class of a fibre. It is well known that two of the four torus invariant divisors on $X$ lie in the class of $F$ and the remaining two are in the classes of $E$ and $E^{\prime}$. Note that all torus invariant divisors but $E^{\prime}$ are nef. Therefore to satisfy property $P_{k}$, a line bundle $L \simeq \mathcal{O}_{X}(a F+b E)$ must satisfy that $a \geq k r$ and $b \geq 1$. Indeed, big and nef (resp. ample) bundles on $X$ are given by $a, b \geq 0$ (resp. $>0$ ) (see [BC13, Theorem 2.4(3)] or [CLS11, Example 6.1.16]) and property $P_{k}$ above mandates $L+k E^{\prime}$ to be ample. Then for a reduced curve $D$ in the class $(c, d)$ we have,

$$
H^{i}\left(X, \mathcal{O}_{X}((k+1)(c+r)-2,(k+1) d-1)\right)=0 \text { for all } i>0 .
$$

Applications. As an application we address the classical problem of finding the number of conditions imposed on certain spaces of hypersurfaces by the isolated singular points on a given singular hypersurface. 
Corollary B. Let $D$ be a reduced effective divisor on a smooth projective toric variety $X$ of dimension $n$. Let $S_{m}(D)$ denote the set of all isolated singular points in $D$ with multiplicity at least $m$. Then $S_{m}(D)$ imposes independent conditions on the hypersurfaces in

$$
H^{0}\left(X, \mathcal{O}_{X}\left(\left(\left\lfloor\frac{n}{m}\right\rfloor+1\right) D-\sum_{i} D_{i}\right) \otimes L\right)
$$

for any line bundle $L$ satisfying property $P_{\left\lfloor\frac{n}{m}\right\rfloor}$.

In particular, when $X=\mathbb{P}^{n}$ we recover [MP16, Corollary H]. We discuss some examples in $\S 4$.

We can extend this analysis to the study of $(j-1)$-jets along $S_{m}(D)$, just as was done in [MP16, Corollary 27.3]. Recall that a line bundle $M$ is said to separate $(j-1)$-jets along a set of points $S \subset X$ if the map

$$
H^{0}(X, M) \longrightarrow \bigoplus_{p \in S} \mathcal{O}_{X} / \mathfrak{m}_{p}^{j}
$$

is surjective. In particular, $S$ imposes independent conditions on $H^{0}(X, M)$ means that $M$ separates 0 -jets along $S$. For $m \geq 3$, define

$$
k_{m, j}= \begin{cases}\left\lceil\frac{j+n-m}{m}\right\rceil & \text { if } j \leq m-1 \\ \left\lceil\frac{j+n-m}{m-2}\right\rceil & \text { if } j \geq m\end{cases}
$$

Then we obtain the following:

Corollary C. With the assumptions and notation in Corollary B, for $m \geq 3$ the space of hypersurfaces

$$
H^{0}\left(\mathcal{O}_{X}\left(\left(k_{m, j}+1\right) D-\sum_{i} D_{i}\right) \otimes L\right)
$$

separate $(j-1)$-jets along $S_{m}(D)$ for any line bundle $L$ satisfying the property $P_{k_{m, j}}$.

Acknowledgements. I am greatly thankful to my advisor Mihnea Popa for suggesting the problem and for his constant support and encouragement throughout the preparation of this paper. I would also like to thank Mircea Mustaţă for various discussions regarding toric varieties and Emanuel Reinecke for various comments on an earlier version of this draft. Lastly, I would like to thank both Mircea Mustaţă and Mihnea Popa for spurring my interest in this area with their series of articles on Hodge ideals.

\section{Preliminaries}

2.1. Eagon-Northcott complexes. We first discuss the general theory behind the construction of a Koszul-type complex that will be used in the proof of Theorem A. The main reference for this section is [Laz04, Appendix B]. The following lemma is surely well-known to experts, nonetheless we include the proof for completeness.

Consider a short exact sequence of vector bundles on a variety $X$ :

$$
0 \rightarrow \Omega \rightarrow \mathcal{E} \rightarrow \mathcal{F} \rightarrow 0
$$

such that $\operatorname{rk} \mathcal{E}=e, \operatorname{rk} \mathcal{F}=f$ with $e>f$. Hence, $\operatorname{rk} \Omega=e-f$. We denote $\bigwedge^{p} \Omega=: \Omega^{p}$. We then have the following resolution of $\Omega^{p}$ in terms of $\mathcal{E}$ and $\mathcal{F}$ (see also [loc. cit. Appendix B.2]): 
Lemma 2.1. The following complex is exact and gives a resolution for $\Omega^{p}$ :

$$
0 \rightarrow \bigwedge^{e} \mathcal{E} \otimes S^{e-p-f} \mathcal{F}^{\vee} \otimes\left(\bigwedge^{f} \mathcal{F}\right)^{\vee} \rightarrow \cdots \rightarrow \bigwedge^{p+f+j} \mathcal{E} \otimes S^{j} \mathcal{F}^{\vee} \otimes\left(\bigwedge^{f} \mathcal{F}\right)^{\vee} \rightarrow \cdots \rightarrow \bigwedge^{p+f} \mathcal{E} \otimes\left(\bigwedge^{f} \mathcal{F}\right)^{\vee}
$$

where $S^{j} \mathcal{F}^{\vee}$ denotes the $j^{\text {th }}$ symmetric power of $\mathcal{F}^{\vee}$.

Proof. Since the morphism $\mathcal{E} \rightarrow \mathcal{F}$ is a map of vector bundles, we obtain by [Laz04, Theorem B.2.2.], that the $p^{\text {th }}$ Eagon-Northcott complex $\left(E N_{p}\right)$

$$
\begin{aligned}
0 \rightarrow \bigwedge^{e} \mathcal{E} \otimes S^{e-p-f} \mathcal{F}^{\vee} \otimes(\bigwedge \mathcal{F})^{\vee} \rightarrow \cdots & \rightarrow \bigwedge^{p+f+j} \mathcal{E} \otimes S^{j} \mathcal{F}^{\vee} \otimes\left(\bigwedge^{f} \mathcal{F}\right)^{\vee} \rightarrow \cdots \rightarrow \bigwedge^{p+f} \mathcal{E} \otimes\left(\bigwedge^{f} \mathcal{F}\right)^{\vee} \rightarrow \\
& \rightarrow \bigwedge^{p} \mathcal{E} \stackrel{p}{\rightarrow} \bigwedge^{p-1} \mathcal{E} \otimes \mathcal{F} \rightarrow \cdots \rightarrow E \otimes S^{p-1} \mathcal{F} \rightarrow S^{p} \mathcal{F} \rightarrow 0
\end{aligned}
$$

is exact.

To determine the kernel of $\phi: \bigwedge^{p} \mathcal{E} \rightarrow \bigwedge^{p-1} \mathcal{E} \otimes \mathcal{F}$ above, we need to analyse the construction of $\left(E N_{p}\right)$. Consider the projective bundle $\mathbb{P}(\mathcal{F}):=\underline{\operatorname{Proj}}\left(\bigoplus_{i} S^{i} \mathcal{F}\right)$ with the map $\pi: \mathbb{P}(\mathcal{F}) \rightarrow X$ and the map of vector bundles

$$
\pi^{*} \mathcal{E}(-1) \rightarrow \mathcal{O}_{\mathbb{P}(\mathcal{F})} .
$$

Denote by $K:=\operatorname{Ker}\left(\pi^{*} \mathcal{E}(-1) \rightarrow \mathcal{O}_{\mathbb{P}(\mathcal{F})}\right)$ the kernel vector bundle. Then

$$
\operatorname{Ker}(\phi)=\operatorname{Ker}\left(\pi_{*} \bigwedge^{p} \pi^{*} \mathcal{E} \stackrel{\phi}{\rightarrow} \pi_{*}^{p-1} \bigwedge^{*} \pi^{*} \mathcal{E}(1)\right) \simeq \pi_{*}\left(\bigwedge^{p} K\right)(p) .
$$

But the last term is isomorphic to $\Omega^{p}$. Indeed, snake lemma applied to the following exact grid:

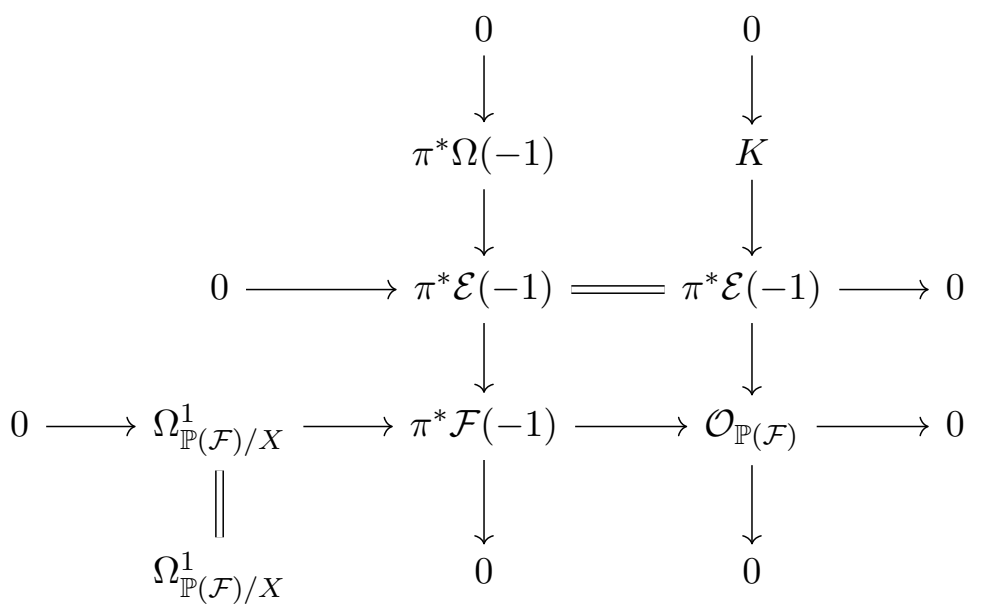

gives us the short exact sequence

$$
0 \rightarrow \pi^{*} \Omega \rightarrow K(1) \rightarrow \Omega_{\mathbb{P}(\mathcal{F}) / X}(1) \rightarrow 0 .
$$

(In the above diagram, the horizontal short exact sequence is the relative Euler sequence and the middle vertical sequence is the pullback of (3) under a smooth morphism.)

Now for a fixed $p$ we have a filtration

$$
\left(\bigwedge^{p} K\right)(p)=F_{0} \supseteq F_{1} \supseteq \cdots \supseteq F_{p+1}=0
$$

such that

$$
F_{k} / F_{k+1}=\pi^{*} \Omega^{k} \otimes \Omega_{\mathbb{P}(\mathcal{F}) / X}^{p-k}(p-k) .
$$


We assert that

$$
\pi_{*} \Omega_{\mathbb{P}(\mathcal{F}) / X}^{p-k}(p-k)=0 \text { for all } p-k \geq 0 .
$$

Granted this, $\pi_{*} F_{k+1} \simeq \pi_{*} F_{k}$ for all $k \leq p$. In particular, $\Omega^{p} \simeq \pi_{*} F_{p} \simeq \pi_{*} F_{0} \simeq \pi_{*}\left(\bigwedge^{p} K(p)\right)$.

The assertion follows from the Koszul resolution of $\pi_{*} \Omega_{\mathbb{P}(\mathcal{F}) / X}^{k}(k)$, namely:

$$
\bigwedge^{f} \pi^{*} \mathcal{F}(-k+f) \rightarrow \cdots \rightarrow \bigwedge^{k+1} \pi^{*} \mathcal{F}(-1) \rightarrow \Omega_{\mathbb{P}(\mathcal{F}) / X}^{k}(k) \rightarrow 0
$$

and the equality $R^{i} \pi_{*} \bigwedge^{k+j} \pi^{*} \mathcal{F}(-j)=0$ for all $i$ and for all $1 \leq j \leq f-1$ (see [Har77, Exer. III.8.4.(c)]).

We apply the above lemma to smooth toric varieties to obtain a resolution of $\Omega_{X}^{p}$.

Lemma 2.2. Let $X$ be a smooth toric variety with torus invariant divisors $D_{i}, i=1, \ldots, d$ then, $\Omega_{X}^{p}$ admits the following Koszul-type resolution:

$$
\begin{aligned}
0 \rightarrow \bigoplus \omega_{X} \rightarrow \cdots \rightarrow \bigoplus \bigoplus_{\sigma \in S_{n-p-j}} \omega_{X}\left(D_{\sigma_{1}}+\cdots+D_{\sigma_{n-p-j}}\right) \rightarrow \\
\cdots \rightarrow \bigoplus \bigoplus_{\sigma \in S_{n-p}} \omega_{X}\left(D_{\sigma_{1}}+\cdots+D_{\sigma_{n-p}}\right) \rightarrow \Omega_{X}^{p} \rightarrow 0 .
\end{aligned}
$$

Here $S_{j}$ is the set of all ordered sequences of length $j$ in $\{1, \ldots, d\}$.

Proof. Lemma 2.1 applied to the short exact sequence (1) gives the following resolution:

$$
\begin{aligned}
0 \rightarrow \bigoplus \bigwedge_{i=1}^{d}\left(\bigoplus_{X}^{d} \mathcal{O}_{X}\left(-D_{i}\right)\right) \rightarrow \cdots \rightarrow \bigoplus \bigwedge^{d-n+p+j}\left(\bigoplus_{i=1}^{d} \mathcal{O}_{X}\left(-D_{i}\right)\right) \rightarrow \\
\cdots \rightarrow \bigoplus \bigwedge^{d-n+p}\left(\bigoplus_{i=1}^{d} \mathcal{O}_{X}\left(-D_{i}\right)\right) \rightarrow \Omega_{X}^{p} \rightarrow 0 .
\end{aligned}
$$

Since $\omega_{X} \simeq \mathcal{O}_{X}\left(-\sum_{i=0}^{d} D_{i}\right)$ [CLS11, Theorem 8.2.3.], we can rewrite each term in the above long exact sequence as the corresponding one from the long exact sequence in the statement.

2.2. Preliminaries on Hodge ideals. Let $D$ be a reduced effective divisor on a smooth variety $X$. Hodge ideals associated to $D$ arise as a measure of the deficit between the Hodge filtration and the pole-order filtration on the Hodge module $\mathcal{O}_{X}(* D):=\bigcup_{k>0} \mathcal{O}_{X}(k D)$. If $D$ is smooth, one has $F_{k} \mathcal{O}_{X}(* D)=\mathcal{O}_{X}((k+1) D)$, in other words $I_{k}(D)=\mathcal{O}_{X}$.

Similarly, when $D$ is an effective $\mathbb{Q}$-divisor and $H$ is an integral divisor such that $D=(1-\beta) H$ with $\beta<1$, one can define Hodge ideals associated to $D$. However in this case one needs to consider the rank 1 free $\mathcal{O}_{X}\left(* D_{\text {red }}\right)$-module generated by $h^{\beta}$, where $h$ is a local equation of $H$. It turns out that these local $\mathcal{D}$-modules appear as direct summand of certain Hodge modules, namely the pushforward of the sheaf of meromorphic functions with poles along the preimage of $D_{\text {red }}$, under some local cyclic cover along $h$ (see [MP18, Lemma 2.6]). Therefore one can make sense of a filtration on $\mathcal{O}_{X}\left(* D_{\text {red }}\right) h^{\beta}$ induced from the Hodge filtration of the ambient Hodge module. Moreover this filtration coincides with the Hodge filtration on $\mathcal{O}_{X}(* D)$ whenever $D$ is reduced. We refer the reader to [MP18] for details.

Definition 2.3. Let $D, H$ and $h$ be as before. Then for an integer $k \geq 0$, the $k^{\text {th }}$-Hodge ideal associated to $D$ is defined by the following equation:

$$
F_{k} \mathcal{O}_{X}\left(* D_{\text {red }}\right) h^{\beta}=I_{k}(D) \otimes \mathcal{O}_{X} \mathcal{O}_{X}\left(k D_{\text {red }}+H\right) h^{\beta} .
$$


The filtration $F$ coincides with the Hodge filtration when $D$ is reduced.

Remark 2.4. The local $\mathcal{D}$-modules $\mathcal{O}_{X}\left(* D_{\text {red }}\right) h^{\beta}$ do not, in general, glue to a global object. However by [MP18, Remark 2.14], $I_{k}(D)$ is independent of the choice of $h$ and $\beta$ and hence is defined globally. The additional assumptions on $D$, in the statement of Theorem A, ensure a global object. In other words, when we have $\beta=\frac{\ell-1}{\ell}$ and $\mathcal{O}_{X}(H) \simeq M^{\otimes \ell}$ for some integer $\ell$ and some line bundle $M$ on $X$, the local $\mathcal{D}$-modules $\mathcal{O}_{X}(* D) h^{\beta}$ glue. Following [MP18, §5], we denote this global $\mathcal{D}$-module by $\mathcal{M}_{1}$. As an $\mathcal{O}_{X}$-module, we have

$$
\mathcal{M}_{1} \simeq M \otimes_{\mathcal{O}_{X}} \mathcal{O}_{X}\left(* D_{\text {red }}\right) .
$$

For the proof of Theorem A that we present, it is crucial that we work with a globally defined $\mathcal{D}$-module.

The induced Hodge filtration on $\mathcal{M}_{1}$ is given by (see, for instance, the proof of [MP18, Theorem 12.1])

$$
F_{k} \mathcal{M}_{1} \simeq M\left(-D_{\text {red }}\right) \otimes_{\mathcal{O}_{X}} \mathcal{O}_{X}\left((k+1) D_{\text {red }}\right) \otimes I_{k}(D)
$$

to which one can associate its filtered deRham complex $\operatorname{DR}\left(\mathcal{M}_{1}\right)$.

Definition 2.5 (The de-Rham complex). For any non-negative integer $k$, the $k^{\text {th }}$ filtered piece of the de-Rham complex of $\mathcal{M}_{1}$ is defined by

$$
F_{k} \operatorname{DR}\left(\mathcal{M}_{1}\right)=\left[F_{k} \mathcal{M}_{1} \rightarrow \Omega_{X}^{1} \otimes F_{k+1} \mathcal{M}_{1} \rightarrow \cdots \rightarrow \Omega_{X}^{n} \otimes F_{k+n} \mathcal{M}_{1}\right]
$$

and the associated graded complex is given by

$$
g r_{k}^{F} \operatorname{DR}\left(\mathcal{M}_{1}\right)=\left[g r_{k}^{F} \mathcal{M}_{1} \rightarrow \Omega_{X}^{1} \otimes g r_{k+1}^{F} \mathcal{M}_{1} \rightarrow \cdots \rightarrow \Omega_{X}^{n} \otimes g r_{k+n}^{F} \mathcal{M}_{1}\right]
$$

as complexes in degrees $-n, \ldots, 0$.

The higher hypercohomologies of the associated graded complex of Hodge modules satisfy a vanishing statement due to Saito (see [Sai90, §2.g], also see [Sch14], [Pop16]). As a result, one also obtains vanishing statements for the associated graded complex of the filtered direct summand $\mathcal{M}_{1}$. We therefore have:

Lemma 2.6 (Saito vanishing). Let $A$ be an ample line bundle on $X$. Then,

$$
\mathbb{H}^{i}\left(X, g r_{k}^{F} \mathrm{DR}\left(\mathcal{M}_{1}\right) \otimes A\right)=0 \text { for all } i>0, k \geq 0
$$

The following vanishing is a consequence of Artin vanishing (see [MP16, Proposition 22.1] for instance):

Lemma 2.7. Let $X$ be a smooth projective variety and $D$ an effective divisor on $X$ such that $X \backslash D_{\text {red }}$ is affine (when $D$ is ample for instance), then

$$
\mathbb{H}^{i}\left(X, g r_{k}^{F} \operatorname{DR}\left(\mathcal{M}_{1}\right)\right)=0 \text { for all } i>0, k \geq 0 .
$$

\section{Proof of the VANishing theOREM}

We will prove the following:

Variant 3.1 (Variant of Theorem A). With the hypotheses of Theorem A, we have for all integers $k \geq 0$ the following equivalent statements hold.

(1) $H^{i}\left(X, \omega_{X}\left((k+1) D_{r e d}\right) \otimes L \otimes I_{k}(D)\right)=0$ for all $i>0$.

(2) $H^{i}\left(X, \omega_{X} \otimes g r_{k}^{F} \mathcal{M}_{1} \otimes A\right)=0$ for all $i>0$ where $A \otimes M\left(-D_{\text {red }}\right)=L$. 
The proof uses the vanishing Lemmas 2.6 and 2.7, followed by an inductive argument involving a spectral sequence. The base case of the induction is Nadel vanishing for $I_{0}(D)$, which by Remark 1.4 , is the multiplier ideal $\mathcal{J}((1-\epsilon) D)$. The argument is, in spirit, very similar to the proof for projective spaces as in [MP16, Theorem 25.3].

Proof. The filtration on $\mathcal{M}_{1}$ is given by $F_{k} \mathcal{M}_{1} \simeq \mathcal{O}_{X}\left((k+1) D_{\text {red }}\right) \otimes M\left(-D_{\text {red }}\right) \otimes I_{k}(D)$ and therefore we have a short exact sequence:

$$
0 \rightarrow \omega_{X}\left(k D_{\text {red }}\right) \otimes L \otimes I_{k-1}(D) \rightarrow \omega_{X}\left((k+1) D_{\text {red }}\right) \otimes L \otimes I_{k}(D) \rightarrow \omega_{X} \otimes g r_{k}^{F} \mathcal{M}_{1} \otimes A \rightarrow 0
$$

where $A:=L\left(D_{\text {red }}-D\right)$. Since by hypothesis $A$ is ample and $D \sim_{\mathbb{Q}} M$, Nadel vanishing theorem gives,

$$
H^{i}\left(X, \omega_{X}\left(D_{\text {red }}\right) \otimes L \otimes I_{0}(D)\right)=0 \text { for all } i>0 .
$$

Therefore, by induction the above statements are equivalent.

We aim to show the second statement. We have,

$$
g r_{k}^{F} \operatorname{DR}\left(\mathcal{M}_{1}\right)[-n]=\left[g r_{k}^{F} \mathcal{M}_{1} \rightarrow \Omega_{X}^{1} \otimes g r_{k-n+1}^{F} \mathcal{M}_{1} \rightarrow \cdots \rightarrow \Omega^{n-1} \otimes g r_{-1+k}^{F} \mathcal{M}_{1} \rightarrow \omega_{X} \otimes g r_{k}^{F} \mathcal{M}_{1}\right]
$$

in degrees 0 to $n$.

Consider the spectral sequence associated to the complex $C^{\bullet}:=g r_{k}^{F} \operatorname{DR}\left(\mathcal{M}_{1}\right)[-n] \otimes A$ :

$$
E_{1}^{p, q}:=H^{q}\left(X, C^{p}\right) \Longrightarrow \mathbb{H}^{p+q}\left(X, C^{\bullet}\right) .
$$

Now by Saito vanishing (Lemma 2.6), we have $\mathbb{H}^{p+q}\left(X, C^{\bullet}\right)=0$ when $p+q \geq n+1$. Also note that, because of the length of $C^{\bullet}$, the spectral sequence degenerates at level $n+1$, therefore, $E_{n+1}^{p, q} \simeq E_{\infty}^{p, q}$. The latter, $E_{\infty}^{p, q}$, a quotient of $\mathbb{H}^{p+q}\left(X, C^{\bullet}\right)$, also vanishes for $p+q \geq n+1$. As the vanishing statement that we aim for, is $E_{1}^{n, i}=0$ for all $i>0$. It is thus enough to show that $E_{1}^{n, i} \simeq \cdots \simeq E_{n+1}^{n, i}$. In other words, it is enough to show that, $E_{r}^{n-r, i+r-1}=0$ for all $r$ with $1 \leq r \leq n$. Indeed, for each $r \geq 1$, the differential going out of $E_{r}^{n, i}$ maps to $E_{r}^{n+r, i-r+1}$, which is 0 because of the length of $C^{\bullet}$. In fact we will show that,

$$
E_{1}^{n-r, i+r-1}=H^{i+r-1}\left(X, \Omega_{X}^{n-r} \otimes A \otimes g r_{k-r}^{F} \mathcal{M}_{1}\right)=0 .
$$

By Lemma 2.2, $\Omega_{X}^{n-r}$ has the resolution:

$$
\begin{aligned}
0 \rightarrow \bigoplus \omega_{X} \rightarrow \cdots \rightarrow \bigoplus \bigoplus_{\sigma \in S_{r-j}} \omega_{X} \otimes \mathcal{O}_{X}\left(D_{\sigma_{1}}+\cdots+D_{\sigma_{r-j}}\right) \\
\quad \rightarrow \cdots \rightarrow \bigoplus \bigoplus_{\sigma \in S_{r}} \omega_{X} \otimes \mathcal{O}_{X}\left(D_{\sigma_{1}}+\cdots+D_{\sigma_{r}}\right) \rightarrow \Omega_{X}^{n-r} \rightarrow 0
\end{aligned}
$$

where $S_{j}$ is the set of all ordered sequences of length $j$ in $\{1, \ldots, d\}$. Tensoring this long exact sequence with $g r_{k-r}^{F} \mathcal{M}_{1} \otimes A$, we see that it is enough to show,

$$
H^{i+r-1}\left(X, \omega_{X} \otimes A\left(D_{\sigma_{1}}+\cdots+D_{\sigma_{j}}\right) \otimes g r_{k-r}^{F} \mathcal{M}_{1}\right)=0
$$

for all $j \leq r$. But this follows by induction, since $L^{\prime}:=L\left(D_{\sigma_{1}}+\cdots+D_{\sigma_{j}}\right)$ with $j \leq r$ satisfies property $P_{k-r}(D)$ by our hypotheses.

Further if $D$ and the $D_{i}$ 's are ample, we can take $A \simeq \mathcal{O}_{X}$. In this case, to begin the induction, we resort to the Artin-type vanishing in Lemma 2.7 and obtain:

$$
\mathbb{H}^{p+q}\left(X, C^{\bullet}\right)=0 \text { for all } p+q \geq n+1 .
$$

The latter steps of the induction use Saito vanishing (Lemma 2.6) with $A^{\prime}:=\mathcal{O}_{X}\left(D_{\sigma_{1}}+\cdots+D_{\sigma_{j}}\right)$, which are automatically ample due to the ampleness assumption on the $D_{i}$ 's. The remaining argument follows the first part of the proof verbatim. 


\section{Applications}

In this section the divisor $D$ is reduced and effective.

We now apply Theorem A to deduce Corollaries B and C. In [MP16, Corollary 27.2] similar results for projective spaces were deduced. The key point is to understand how deep the Hodge ideals sit inside the maximal ideal of an isolated singular point. This has already been studied in [Theorem E, Corollary 19.4 loc. cit.]. Without proof we collect their results in the following:

Lemma 4.1. Let $D$ be a reduced hypersurface on a smooth variety $X$ of dimension $n$. Let $x$ be an isolated singular point of $D$ with mult $_{x}(D)=m$. Let $k$ be an integer such that $\frac{n}{k+1}<m<\frac{n}{k}$, then

$$
I_{k}(D) \subseteq \mathfrak{m}_{x}^{(k+1) m-n} .
$$

Further if $m \geq \frac{n}{k}$, then

$$
I_{k}(D) \subseteq \mathfrak{m}_{x}^{\ell} \quad \text { where } \ell=\max \{m-1,(k+1)(m-2)-n-2\}
$$

Proof of Corollary B. Recall that $S_{m}(D)$ is the set of all isolated singular points on $D$ with multiplicity at least $m$. From Lemma 4.1 we know that for $k=\left\lfloor\frac{n}{m}\right\rfloor$ and for all $p \in S_{m}(D), I_{k}(D)_{p} \subseteq \mathfrak{m}_{p}$. Therefore for a line bundle $L$ satisfying $P_{k}$, we have:

$$
H^{1}\left(X, \mathcal{O}_{X}\left(\left(\left\lfloor\frac{n}{m}\right\rfloor+1\right) D-\sum_{i} D_{i}\right) \otimes L \otimes I_{k}(D)\right)=0 .
$$

This yields the following surjection

$$
H^{0}\left(X, \mathcal{O}_{X}\left(\left(\left\lfloor\frac{n}{m}\right\rfloor+1\right) D-\sum_{i} D_{i}\right) \otimes L\right) \longrightarrow \bigoplus_{p \in S_{m}} \mathcal{O}_{X} / \mathfrak{m}_{p}
$$

thereby proving the statement.

Note that the choice of $k$ is optimal for this proof. For example, by [MP16, Theorem D], when $p$ is an ordinary isolated singular point of $D$ and $k \leq\left\lfloor\frac{n}{m}\right\rfloor-1$, locally around $p$ we have

$$
I_{k}(D)_{p} \simeq \mathcal{O}_{X, p}
$$

The proof of separation of $(j-1)$-jets follows similarly. For the sake of completeness, we include it here.

Proof of Corollary C. First note that $I_{k_{m, j}}(D) \subseteq \mathfrak{m}_{p}^{j}$ for all $p \in S_{m}(D)$. Indeed, if $m^{\prime}>m$, then $k_{m, j}=k_{m^{\prime}, j^{\prime}}$ for $j^{\prime} \geq j$ and therefore, if mult $_{p^{\prime}} D=m^{\prime}>m$ for some point $p^{\prime} \in S_{m}(D)$, then $I_{k_{m, j}}(D) \subseteq \mathfrak{m}_{p^{\prime}}^{j^{\prime}} \subseteq \mathfrak{m}_{p^{\prime}}^{j}$. Now for a line bundle $L$ satisfying $P_{k_{m, j}}$, we have the vanishing in Theorem A for $I_{k_{m, j}}(D)$ and therefore we obtain a surjection:

$$
H^{0}\left(X, \mathcal{O}_{X}\left(\left(k_{m, j}+1\right) D-\sum_{i} D_{i}\right) \otimes L\right) \longrightarrow \bigoplus_{p \in S_{m}} \mathcal{O}_{X} / \mathfrak{m}_{p}^{j}
$$

Hence the Corollary.

\section{Examples.}

(1) Let $X=\mathbb{P}^{n_{1}} \times \mathbb{P}^{n_{2}}$ and denote $n:=n_{1}+n_{2}$. Let $D$ be a reduced effective divisor of type $(c, d)$. Since the toric divisors on $X$ are nef, any ample line bundle, and in particular $L=\mathcal{O}_{X}(1,1)$, satisfies property $P_{k}$ for for all $k$. Therefore, $S_{m}(D)$ imposes independent conditions on the space of hypersurfaces

$$
H^{0}\left(X,\left(\left(\left\lfloor\frac{n}{m}\right\rfloor+1\right) c-n_{1},\left(\left\lfloor\frac{n}{m}\right\rfloor+1\right) d-n_{2}\right)\right) .
$$


In particular, isolated singular points on a surface in the class $(c, d)$ on $X=\mathbb{P}^{2} \times \mathbb{P}^{1}$ impose conditions on $\left|\mathcal{O}_{X}(2 c-2,2 d-1)\right|$. This can be compared with the Severi-type bound as in [PW06, Main Theorem] for $\mathbb{P}^{3}$, namely isolated singular points on a surface of degree $d$ in $\mathbb{P}^{3}$ impose independent conditions on hypersurfaces of degree at least $2 d-5$.

(2) Let $X=F_{r}$, the Hirzebruch surface with $r \geq 0$, as in Example 3 from the introduction. Note that the line bundle $L=\mathcal{O}_{X}((r F+E))$ satisfies $P_{1}$. Moreover, we have $K_{X} \sim$ $(r-2) F-2 E$. Then for any reduced effective singular divisor $D$ in the class $(c, d), S_{2}(D)$ imposes independent conditions on the space of hypersurfaces

$$
H^{0}\left(X, \mathcal{O}_{X}((2(c+r)-2) F+(2 d-1) E)\right) .
$$

\section{REFERENCES}

[BC13] A. Bertram and I. Coskun, "The birational geometry of the Hilbert scheme of points on surfaces" Birational geometry, rational curves, and arithmetic, Simons Symp. p.15-55 Springer, Cham. 2013. MR:3114922. DOI: 10.1007/978-1-4614-6482-2-2. 2

[CLS11] D. A. Cox, J. B. Little and H. K. Schenk, Toric varieties Graduate Studies in Mathematics, Vol. 124 American Mathematical Society, Providence, RI, 2011 ISBN: 978-0-8218-4819-7. MR:2810322. DOI:10.1090/gsm/124. 2, 5

[Har77] R. Hartshorne, Algebraic geometry. Grad. Texts in Math., Vol. 52. New York-Heidelberg: Springer-Verlag, 1977. DOI: 10.1007/978-1-4757-3849-0. MR: 0463157. 5

[Laz04] R. Lazarsfeld, Positivity in algebraic geometry. I. Classical setting: line bundles and linear series. Ergeb. Math. Grenzgeb. (3), Vol. 48. Berlin: Springer-Verlag, 2004. DOI: 10.1007/978-3-642-18808-4. MR: 2095471. 3, 4

[MP16] M. Mustaţă and M. Popa, "Hodge ideals." 2016. to appear in Memoirs of the AMS. arXiv:1605.08088 [math.AG]. 1, 2, 3, 6, 8

[MP18] M. Mustaţă and M. Popa, "Hodge ideals for $\mathbb{Q}$-divisors I - birational approach." 2018. arXiv:1807.01932. $1,2,5,6$

[PW06] J. Park and Y. Woo, "A remark on hypersurfaces with isolated singularities." Manuscripta Math. 121, 2006. p. 451-456. DOI: 10.1007/s00229-006-0047-1. MR:2283472. 9

[Pop16] M. Popa, "Kodaira-Saito vanishing and applications." L'Enseignement Mathématique Vol. 62, 2016. 1-2, p. 49-89, DOI: 10.4171/LEM/62-1/2-5. MR: 3605809. 6

[Sai90] M. Saito, "Mixed Hodge modules." Publ. Res. Inst. Math. Sci., Kyoto University. Research Institute for Mathematical Sciences. Publications, Vol. 26, 1990, 2, p. 221-333. DOI: 10.2977/prims/1195171082. MR:1047415. 6

[Sch14] C. Schnell, "On Saito's vanishing theorem." Mathematical Research Letters, vol. 23, 2016. 2, p. $499-527$. DOI: 10.4310/MRL.2016.v23.n2.a10. MR:3512896. 6

Department of Mathematics, Northwestern University, Evanston, IL 60208-2730, USA

E-mail address: ydutta@math.northwestern.edu

URL: http://www.math.northwestern.edu/ ydutta/ 